\title{
Comparing complete and partial classification for identifying customers at risk
}

\author{
Josée M.M. Bloemer ${ }^{\mathrm{a}, *}$, Tom Brijs $^{\mathrm{b}}$, Koen Vanhoof ${ }^{\mathrm{b}}$, Gilbert Swinnen ${ }^{\mathrm{b}}$ \\ ${ }^{a}$ Nijmegen School of Management, University of Nijmegen, P.O. Box 9108, NL-6500 HK Nijmegen, The Netherlands \\ ${ }^{\mathrm{b}}$ Limburgs Universitair Centrum, Universitaire Campus, 3590 Diepenbeek, Belgium
}

Received 26 April 1999; received in revised form 4 September 2002; accepted 6 September 2002

\begin{abstract}
This paper evaluates complete versus partial classification for the problem of identifying customers at risk. We define customers at risk as customers reporting overall satisfaction, but these customers also possess characteristics that are strongly associated with dissatisfied customers. This definition enables two viable methodological approaches for identifying such customers, i.e. complete and partial classification. Complete classification entails the induction of a classification model to discriminate between overall dissatisfied and overall satisfied instances, where customers at risk are defined as overall satisfied customers who are classified as overall dissatisfied. Partial classification entails the induction of the most prevalent characteristics of overall dissatisfied customers in order to discover overall satisfied customers who match these characteristics. In our empirical work, we evaluate complete and partial classification techniques and compare their performance on both quantitative and qualitative criteria. The intent of the paper is not on proving the superiority of partial classification, but rather to provide an alternative and valuable approach that offers new and different insights. In fact, taking predictive accuracy as the performance criterion, results for this study show the superiority of the complete classification approach. On the other hand, partial classification offers additional insights that complete classification techniques do not offer, i.e. it offers a rule-based description of criteria that lead to dissatisfaction for locally dense regions in the multidimensional instance space.
\end{abstract}

(C) 2003 Elsevier Science B.V. All rights reserved.

Keywords: Customers at risk; Customer retention; Data mining

\section{Introduction}

Customer loyalty is a well-known and heavily researched topic in the field of marketing. Throughout the years, the main stream of loyalty research has focussed on the willingness of customers to enter and

\footnotetext{
* Corresponding author. Tel.: +31-24-3613018; fax: +31-243611933.

E-mail address: j.bloemer@nsm.kun.nl (J.M.M. Bloemer).
}

to stay in a relationship with the provider of a product or service based on their satisfaction (Anderson, 1998; Anderson \& Mittal, 2000; Anderson \& Sullivan, 1993; Bloemer \& Kasper, 1995; Bolton, 1998; Bowman \& Narayandas, 2001; Day, 1977; Dver, 2001; Enew \& Brinks, 1996; Garfein, 1987; Hirschman, 1970; Homburg \& Giering, 2001; Jones, Mothersbaugh, \& Beatty, 2000; Oliver, 1980, 1997; Oliver \& Swan, 1989a, 1989b; Swan, Trawick, \& Carroll, 1982).

However, some scholars have pinpointed the fact that more attention needs to be paid to those customers 
who, although they are loyal at the moment, might have a higher propensity to leave than others and are somehow less willing to stay in the relationship. In general terms, Berry (1995) asks for additional attention on how to reduce customer defection. To the best of our knowledge, there are only two studies available in the literature that address this issue more specifically.

In 1984, Gensch showed that supplier loyalty, i.e. the current relative strength of customer preference for a given supplier, is a key dimension to consider in allocating marketing effort. With the help of a logit model, Gensch segments the total market on the current brand loyalty dimension. The different resulting segments vary in their degree of loyalty towards various suppliers. Two groups are of major interest. The 'switchable' customer, who currently perceives the firm to be very close to its first choice suppliers, and a 'competitive' customer, who, although he currently perceives our firm as his first choice, perceives other suppliers as being very close to our firm. Targeting these two groups with a so-called 'missionary' approach and allocating additional and specific marketing resources towards these groups produced impressive positive sales results. Gensch herewith shows that distinguishing between loyal customers and potential switchers and addressing them differently with a retention programme could be a fruitful approach in protecting potential switchers from leaving and spending even more.

In a recent study of Ganesh, Arnoldt, and Reynolds (2000), a distinction is made between three segments of the current customers of a retail banking service according to whether or not they have switched from other service providers in the past and, if so, whether the switching was based on dissatisfaction or not (e.g. job rotation). The three distinguished segments are called 'dissatisfied switchers', 'satisfied switchers' and 'stayers'. The study clearly shows that the three different segments differ significantly in terms of their satisfaction with the current service provider and their loyalty towards that service provider. The results indicate that 'dissatisfied switchers' are the most satisfied customers and are most likely to engage in active loyalty behaviour, whereas 'stayers' who exhibit higher satisfaction than 'satisfied switchers' but lower satisfaction than the 'dissatisfied switchers', are more likely to exhibit passive loyalty behaviour. 'Satisfied switchers' are the least satisfied customers and fall between the other two groups in terms of both active and passive loyalty behaviour. This study therefore also makes clear that it is worthwhile to recognise the heterogeneity inherent in a firm's customer base and to treat these segments differently with regard to strategic investments in loyalty.

Nevertheless, before one can act upon specific customer segments that seem to be specifically vulnerable in terms of customer defection, one has to identify these customers.

The key contribution of this article is showing the potential of complete and partial classification techniques for identifying such vulnerable customer segments. More specifically, an empirical comparison of the techniques will be made along several quantitative and qualitative criteria, based on a large data set from a Belgian bank. We will call customers that are especially vulnerable in terms of customer defection 'customers at risk'. Customers at risk are those customers who state to be satisfied on an overall level, but who nevertheless possess some other characteristics similar to customers reporting overall dissatisfaction. In this sense, customers at risk form an important — but hidden-segment that deserves special attention in terms of loyalty and retention management. Indeed, because of their resemblance to dissatisfied customers in terms of their (dis)satisfaction with, for instance, specific service attributes and/or sociodemographic characteristics, customers at risk might have a higher expected probability of becoming overall dissatisfied in the near future. As a result, this might lead to a decrease in loyalty in terms of actual defection (Gensch, 1984), diminishing active loyalty (Ganesh et al., 2000) or a diminishing purchase intention, less positive word of mouth, an increase in price-sensitivity and less willingness to complain to the organisation itself (Zeithaml, Berry, \& Parasuraman, 1996). In other words, customers at risk are customers that might have a higher propensity to become overall dissatisfied in the near future and to act in accordance with that dissatisfaction. Although this link is not being studied in this paper, the identification of these customers might therefore be seen as an early warning signal for those customers that might have a higher propensity to leave than others and are somehow less willing to stay in a relationship.

The remainder of this paper is organised as follows. Firstly, we will elaborate on the two methodo- 
logical approaches to the problem of identifying customers at risk. For both approaches, a number of techniques will be discussed and empirically evaluated on different criteria on real customer satisfaction data. In addition, validation will be carried out on separate testing data. The final section will be reserved for conclusions.

\section{Alternative methodological approaches}

\subsection{Approach 1: complete classification}

In the complete classification approach, a classification model, which discriminates between overall dissatisfied (positive) and overall satisfied (negative) instances, is induced on the data. The term complete classification refers to models covering all instances and all classes in the data. Furthermore, a distinction can be made between function-based techniques (e.g. logistic regression, mixture regression) that use a classification function to predict the class membership of instances and rule-based techniques (e.g. CART, C5.0) that use classification rules for prediction. Customers at risk can then be defined as overall satisfied instances that are misclassified by the model as 'overall dissatisfied', i.e. false positives (FP).

Since most of the readers are familiar with logistic regression, we will only describe C5.0, CART, and latent class regression.

\subsubsection{C5.0}

C5.0 is the latest version of the C4.5 classification tree algorithm by Quinlan (1993). C5.0 constructs classification trees by recursively splitting the instance space into smaller subgroups until the subgroup contains only instances from the same class (a pure node), or the subgroup contains instances from different classes (unpure) but the number of instances in that node is too small to be split further. Typically, the tree is allowed to grow its full size after which it is pruned back upwards in order to increase its generalisation power on unseen data and to reduce potential overfitting. The decision to split on a specific attribute and attribute value is determined by the gain or gain ratio criterion and is based on the principles of information entropy. In contrast to CART, which produces binary splits on the attributes, C5.0 creates multiple branches per split, i.e. one for each different attribute value for a discrete attribute, or in the case of a continuous attribute, one for each interval after discretization of that attribute.

\subsubsection{CART}

In CART (Breiman, Friedman, Olshen, \& Stone, 1984), the construction of the tree classifier is also carried out by recursively splitting the instance space in smaller subparts, however, in this case, until some stopping criterion is achieved. The goodness of the split is defined to be the decrease in impurity (measured by the Gini index of diversity) of the parent node versus the child nodes as a result of a binary split. The growing process of the tree is terminated as soon as the decrease in impurity, by further splitting a specific subspace of instances, falls below a user-defined threshold. In that case, the node is called a terminal or leaf node.

\subsubsection{Mixture regression}

The model estimated in this study belongs to the class of generalized mixture regression models (Wedel \& Kamakura, 1999) where the dependent variable is assumed to be distributed according to a particular density distribution (in this case binomial) and where the expectation of the dependent variable is modelled as a function of a set of independent variables (in this case a logit link). However, since the data are assumed to arise from a number of a priori unknown segments (latent classes) that are mixed in unknown proportions, the objective is to simultaneously 'unmix' the observations and to estimate the regression function within each segment.

Information based criteria, such as CAIC (Eisenblätter \& Bozdogan, 1988) and BIC (Schwarz, 1978), are used to select the most appropriate model. These are goodness-of-fit measures that take into account model parsimony, i.e. the increased fit of a more complex model is weighed against the additional number of parameters needed to produce that fit. The quality of the produced models can be further evaluated by examining the level of separation between the produced segments, as measured by the entropy statistic (Es).

\subsection{Approach 2: partial classification}

Partial classification refers to the discovery of models that show characteristics of the data classes, but may 
not cover all classes and all instances of any given class. Since the objective is to learn descriptive rules that are individually accurate, their main goal is not to predict class values, but rather to discover the necessary or most prevalent characteristics of some or all of the data classes (Ali, Manganaris, \& Srikant, 1997). Especially in domains where most of the attribute values are missing, or the class distributions are very skewed and the user is especially interested in understanding some low frequency class, partial classification can be preferred over complete classification.

Customers at risk are then identified in a slightly different way. Firstly, characteristics are generated (in terms of frequently co-occurring attribute-value combinations) that are prevalent within the group of overall dissatisfied customers. Given these frequently co-occurring attribute-value combinations, customers in the other group (overall satisfied) who have similar characteristics, are selected. We call the latter group customers at risk because, although customers in this group report overall satisfaction, they possess characteristics that are prevalent to overall dissatisfied customers. In this paper, we will focus on one particular partial classification technique, i.e. association rules.

\subsubsection{Association rules}

Association rules were first introduced in the context of retail market basket analysis to discover purchase relationships between products in scanner data (Agrawal, Imielinski, \& Swami, 1993). For instance, the association rule IF diapers THEN beer specifies that customers who buy diapers also tend to buy beer with it. The objective of association rules is then to discover all such purchase relationships $(X \Rightarrow Y)$ in the data that satisfy a minimum (user defined) support and confidence threshold.

The support threshold specifies the minimum proportion of retail baskets in the data in which the items of the association rule must occur together in order for the rule to be frequent. The confidence threshold specifies a minimum threshold on the expected conditional probability of the association rule $P(Y \mid X)$, i.e. the probability that the consequent occurs, given that the antecedent has occurred, in order for the rule to be valid.

The most well-known algorithm to discover association rules is the Apriori algorithm (Appendix B) that was introduced by Agrawal and Srikant (1994).
The application domain of association rules is, however, not restricted to retailing. It has already been used in the context of cross-selling (Anand, Hughes, Bell, \& Patrick, 1997), finding co-occurring medical tests from a health insurance information system (Viveros, Nearhos, \& Rothman, 1996), reducing fall-out in telecommunications systems (Ali et al., 1997), and in this paper to identify latently dissatisfied customers.

\subsection{Comparison of both approaches}

From a conceptual point of view, the difference between complete and partial classification can be illustrated as shown in Fig. 1.

The objective of complete classification is to discriminate between the instances of the positive (dissatisfied) and negative (satisfied) class. In the case of classification trees, this involves the discovery of mutually exclusive and collectively exhaustive (quasi pure) multidimensional cubes in the multidimensional instance space.

In the case of partial classification, however, the objective is to find the necessary or most prevalent characteristics of the target class, i.e. one looks for an accurate description of the target group (overall dissatisfied instances) that is as complete as possible (i.e. which covers as many positive instances as possible).

The difference between both approaches also entails a different way of identifying latently dissatisfied customers. In the case of complete classification, latently dissatisfied customers are situated in the intersection on the left of line number 1 . In the case of partial classification, latently dissatisfied customers are those situated in the intersection on the left of

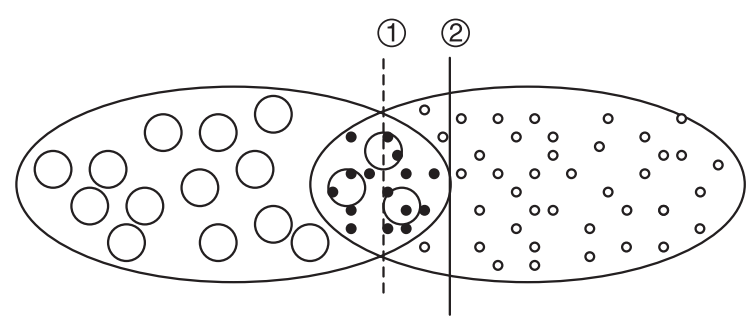

Fig. 1. Manifestly dissatisfied customers are represented as big white dots, whereas small white dots represent manifestly satisfied instances. Latently dissatisfied customers are represented as small black dots. 
line number 2. The reader will have noticed that there exists a relationship between line numbers 1 and 2 . Namely, in the case of complete classification, increasing the cost of false negative errors will lead to a shift of line number 1 into the direction of line number 2. In analogy, line number 2 will be shifted into the direction of line number 1 by pruning rules from the association ruleset, i.e. by softening the constraint that all $(100 \%)$ positive instances should be covered.

\section{Empirical evaluation}

\subsection{Raw data}

The data being used in this study were obtained from a large-scale anonymous customer satisfaction survey carried out by a major Belgian bank in 1996 . In February 1996, a random sample of 7264 customers was obtained, which was used for calibration and, later, in September 1996, an additional sample of 31970 customers was obtained, which was used for testing. The data contains basically three types of questions, i.e. questions probing for satisfaction, questions probing for sociodemographic status (age, marital status, profession, and education), and questions about complaints behaviour (type and number of complaints) and intentions to defect.

Questions related to satisfaction are measured on a five-point rating scale (ranging from strong disagree to strong agree, plus a no opinion option) and can be further subdivided into two types. Firstly there are 16 questions related to satisfaction with specific servicerelated issues of the bank, i.e. with respect to the empathy of the staff (e.g. friendliness, flexibility of personnel), information and communication (e.g. investment advice, telephone helpdesk, etc.), and the practical organisation of the bank office (e.g. opening hours, waiting time). These 16 questions, after recoding (Section 3.2), will become the independent variables in our study.

Secondly, one specific question, again measured on a five-point rating scale, probed for the overall level of (dis)satisfaction of the customer: "Overall, the services offered by the bank meet my expectations". This question will, after recoding, become the dependent variable in this study.

\subsection{Data recoding}

Preliminary analysis of the data revealed a strong bias towards higher levels of satisfaction with only a very small tendency to be very dissatisfied, both on the questions related to satisfaction with specific service items, but also on the target question which probes for overall level of satisfaction.

Therefore, it is suitable to group certain attribute values to obtain more observations per grouped attribute value. For the question probing for overall level of satisfaction, a binary target variable was created, i.e. customers were allocated into two groups: overall satisfied (including agree and strong agree), containing $93.9 \%$ of the observations, and overall dissatisfied (including strong disagree, disagree, and disagree nor agree), containing $6.1 \%$ of the observations.

For the 16 other questions, probing for the level of satisfaction on specific service related items, it is not a priori known which value grouping is optimal for this research. Therefore, we created dummy variables by means of thermometer coding for the independent variables.

\subsection{Empirical evaluation of the different techniques}

\subsubsection{Empirical design}

Multiple classifiers of each type were generated under different parameter settings. In particular for the complete classification approach, C5.0 was carried out with and without misclassification costs, and with and without grouping of symbolic values in the tree (C5.0 GSV). The use of misclassification costs is justified because of the skewed class distributions in the data. Enabling the grouping of symbolic values in C5.0 is done to ensure a fair comparison with CART, which produces binary splits. Just as C5.0, CART was also carried out with and without misclassification costs by adjusting the prior probabilities of the target classes.

For logistic regression and for mixture regression, different models were generated by altering the cut value according to increasing false negative misclassification costs. The idea is as follows. Let dissatisfied $=1$ and satisfied $=2$ and let $C(2 \mid 1)$ be the cost of misclassifying a dissatisfied instance, then an instance is classified as dissatisfied if $P(2 \mid x) * C(1 \mid 2)<$ $P(1 \mid x) * C(2 \mid 1)$. Now suppose $C(2 \mid 1) / C(1 \mid 2)=2$, 
i.e. the cost of misclassifying a positive (dissatisfied) instance costs twice as much as misclassifying a negative (satisfied) instance. Then, it can be calculated from the above formula that the critical cut-off value equals $2 / 3$. According to this logic, different logistic and mixture regression models were generated for misclassification costs ranging from 1 to 9 .

For the mixture regression, a binomial model was specified for the binary dependent variable together with a logit-link function. The analysis was carried out for a range of latent classes (two to five classes) including a random responder class to identify subjects that respond in a random or systematic manner to the measurement scale (i.e. for which only an intercept is estimated).

For the partial classification approach, multiple association rule models were generated according to the following scenario. First, all frequent combinations of attribute values for instances of the target class (overall dissatisfied) were generated given a user-predefined minimum support threshold. The outcome is the set of all combinations of attribute values that appear together in the class of overall dissatisfied customers with frequency exceeding this minimum support threshold (see Fig. 2).

The threshold level of the minimum support parameter is important since setting it too low results in overfitted patterns (e.g. rules describing very few instances) whereas setting this parameter too high results in missing some important customer segments within the target class. Therefore, in our empirical work, different support thresholds (ranging from 5\% to $20 \%$ ) were set to investigate the quality of the resulting classifiers on a separate test set. Secondly, different models of association rules were obtained by modifying the number of rules retained according to a measure of interestingness (Anand et al., 1997). This is necessary because the discovered characteristics may as well be characteristics of the complete dataset because they represent the necessary, but not the sufficient, condition for the membership of the positive example set. Therefore, a filtering system was adopted to select only the interesting or useful rules. In order to rank the rules in terms of interestingness, the following measure was used (Anand et al., 1997):

Interest $_{\mathrm{R}}=S_{\text {Target }}-S_{\text {Total }} / \max \left\{S_{\text {Target }}, S_{\text {Total }}\right\}$

where $S_{\text {Target }}\left(\right.$ resp. $\left.S_{\text {Total }}\right)$ is the support of the rule in the target class (resp. total database). The denominator is introduced to normalise the interestingness between $[-1,+1]$.

\subsubsection{Considerations for fair comparison}

Finding a common performance criterion to compare all the presented techniques is not easy. In order to enable the reader to make a balanced judgement, the suggested classification models will be compared on separate test data along different performance criteria (see Table 2), both quantitative (Section 3.3.3) and qualitative (Section 3.3.4), although not all criteria will be relevant or applicable for each technique. For instance, log likelihood statistics are not applicable (na) for rule-based approaches since classification trees and association rules are non-parametric techniques that do not involve maximum likelihood estimation.

In terms of predictive accuracy, the ROC convex hull graph (Provost \& Fawcett, 1997) is probably the best common quantitative performance criterion. Indeed, the ROC convex hull graph is preferred when class distributions are highly skewed (which is the case for this dataset) or the cost of making errors is not equal.

\begin{tabular}{cc} 
Frequent Itemsets & \\
\hline Frequent Itemset & Support $^{1}$ \\
\hline$\{\mathrm{Q} 9=1\}$ & $42.5 \%$ \\
$\{\mathrm{Q} 11=1\}$ & $33.7 \%$ \\
$\{\mathrm{Q} 9=1, \mathrm{Q} 11=1\}$ & $20 \%$ \\
$\ldots \ldots$ & $\ldots$ \\
\hline
\end{tabular}

\begin{tabular}{lcc}
\hline \multicolumn{1}{l}{ Rules } & & \\
\hline Rule & Support & Confidence \\
\hline $9=1 \&$ Q11=1 $\Rightarrow$ Dissatisfied & $20.0 \%$ & $76.0 \%$ \\
Q5=1 \& Q14=1 $\Rightarrow$ Dissatisfied & $22.9 \%$ & $75.6 \%$ \\
$\cdots$ & $\ldots$ & $\ldots$ \\
\hline
\end{tabular}

Fig. 2. Frequent itemsets and association rules. 
With regard to qualitative criteria, the focus is both on the ease of interpretation and the stability of the model results. Indeed, function-based and rule-based approaches differ in how they represent the discovered relationships between the dependent and independent variables. In this context, special attention will be given to the advantage of the partial classification approach.

Finally, there is the problem of the group size of customers at risk (Section 3.3.5). Indeed, since customers at risk are hidden in the data, we do not exactly know the size of this group beforehand which complicates the process of finding the 'best' model. More specifically, variations of parameter settings for the different classification methods results in different group sizes for the customers at risk. For instance, increasing the cost of false negative classifications will increase the FP rate, which in turn determines the size of the group of customers at risk.

\subsubsection{Quantitative comparison}

The ROC convex hull graph (Provost \& Fawcett, 1997) decouples classifier performance from specific class and cost distributions, and can be used to specify the subset of methods that are potentially optimal under any cost and class distribution assumptions. The true positive rate (TP rate) is plotted on the $Y$ axis and the FP rate on the $X$-axis. One point in the ROC graph (representing one classifier with given parameters) is better than another if it is to the northwest (TP is higher, FP is lower, or both) of the graph.

Fig. 3 illustrates the performance of the different classifiers in this study on the separate testing data. Firstly, one can observe different CART, C5.0, and C5.0 GSV, logistic regression and mixture regression models with increasing false negative costs from 1 to 9. For the classification tree technique, this means that different models have been induced by increasing the false negative, which in turn results in higher TP and

ROC GRAPH

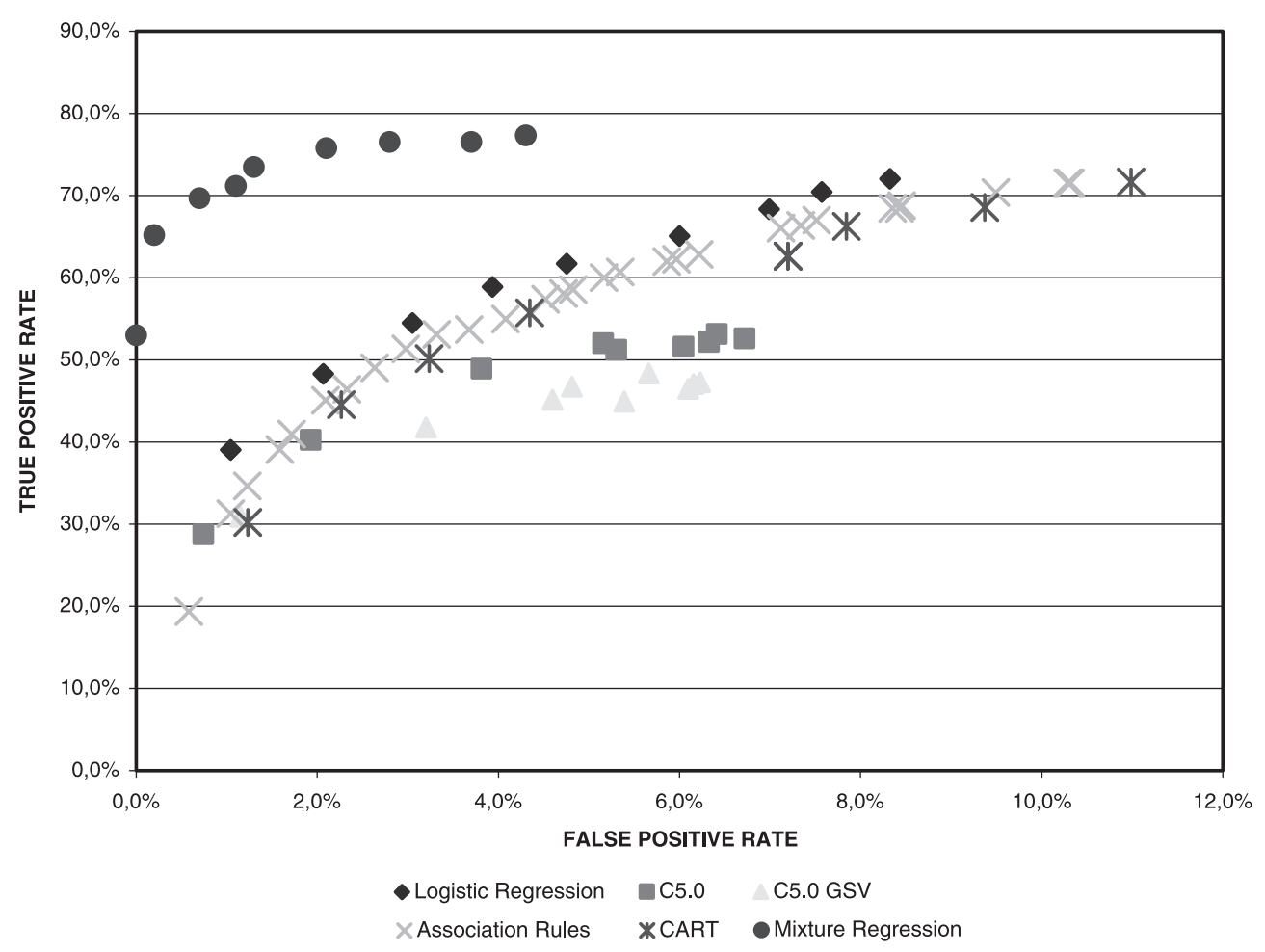

Fig. 3. ROC convex hull graph for different classification models. 
FP rates. For the logistic and mixture regression technique, this means that different models have been induced, each time by increasing the critical cut-off value for classification.

Secondly, the graph also shows the performance of the different association rule rulesets (AR ruleset). The number of rules in the ruleset determines the performance of the model, i.e. the AR ruleset in the bottom left corner of the graph contains only the single most interesting rule. The top right AR ruleset model contains the 29 most interesting rules (see Appendix A) as determined by the interestingness measure (1). The latter ruleset covers more overall dissatisfied instances (higher TP rate) but, because of the addition of less and less 'interesting' rules, the FP rate will increase. AR rulesets containing more rules are not plotted on Fig. 3 since they did not increase the TP rate significantly but only further increased the FP rate.

According to Provost and Fawcett (1997), only when one classifier clearly dominates another over the entire performance space can it be declared better. From Fig. 3, it can be observed that the mixture regression approach is clearly superior on an overall basis. The mixture regression models, shown on the ROC graph, represent a three-segment mixture model with different cutoff values. This three-segment model was selected as the optimal model from a series of mixture models ranging from $k=2$ to 5 segments based on the CAIC and BIC information criteria. The reason for using information criteria to determine the optimal number of segments is motivated by the problem that the likelihood ratio (LR) test statistic can not be used because some regularity conditions for the use of the LR test are violated (Aitkin \& Rubin, 1985). Instead, information criteria have been proposed in the literature as heuristics to select the optimal number of segments in a mixture model. Basically, the idea of these heuristics is to penalize the increase fit (measured by the $\log$ likelihood $L_{k}$ ) of a more complex model with ' $k$ ' segments by the increased number of parameters $\left(d_{k}\right)$ that are needed to estimate this model. The most well-known in this context is the Akaike Information Criterium (AIC) (Akaike, 1974), defined as $\mathrm{AIC}=-2 L_{k}+2 d_{k}$. However, since AIC is dimension inconsistent and tends to overestimate the correct number of segments, the Bayesian Information Criterion $\left(\mathrm{BIC}=-2 L_{k}+\ln (n) d_{k}\right)$ and the Consistent Information Criterion $\left(\mathrm{CAIC}=-2 L_{k}+[\ln (n)+1] d_{k}\right)$ were used since they penalize more heavily for increasing sample size $n$ (Bozdogan, 1987). The optimal number of segments is found there where BIC and CAIC reach a minimum value. The results in Table 1 show that the three-segment solution (including random responder class) is clearly optimal.

Finally, the ROC graph in Fig. 3 shows that the partial classification technique of association rules performs worse than the regression approaches, but better than the rule-based complete classification approaches.

\subsubsection{Qualitative comparison}

The qualitative comparison of the different techniques is carried out on the following criteria: ease of interpretation, heterogeneity and model stability.

With regard to the ease of interpretation of the model results, a distinction can be made between rulebased and function-based models. For rule-based models, such as association rulesets, results are presented to the user in a propositional (IF-THEN) rule format, whereas for function-based models the link between the dependent and independent variables is expressed as a mathematical function. Although the debate which one is most intuitive to interpret is mainly philosophical, it is our opinion that a rulebased approach is probably easier to understand and interpret for a non-expert user in the field, who should use the results of this study to take actions. This can be illustrated by visualizing the association rules in so-called mosaic plots, see Fig. 4.

The mosaic plot shows that if people heavily disagree with question 5 (staff reserves sufficient time for me) and question 14 (staff are flexible enough to solve my questions), there is a very high probability of being overall dissatisfied. This can be verified in rule number 2 in the appendix. Therefore, by visualising the ruleset into mosaic plots, managers can quickly

Table 1

Overview of mixture regression results

\begin{tabular}{lcccc}
\hline \multirow{5}{*}{} & \multicolumn{4}{l}{ Number of latent classes } \\
\cline { 2 - 5 } & 2 & 3 & 4 & 5 \\
\hline LL & -352.53 & -318.8 & -317.1 & -316.1 \\
CAIC & 731 & 681.8 & 696.1 & 711.82 \\
BIC & 728 & 676.8 & 689.1 & 702.82 \\
Es & 0.15 & 0.17 & 0.136 & 0.186 \\
$R^{2}$ & 0.32 & 0.389 & 0.386 & 0.388 \\
\hline
\end{tabular}




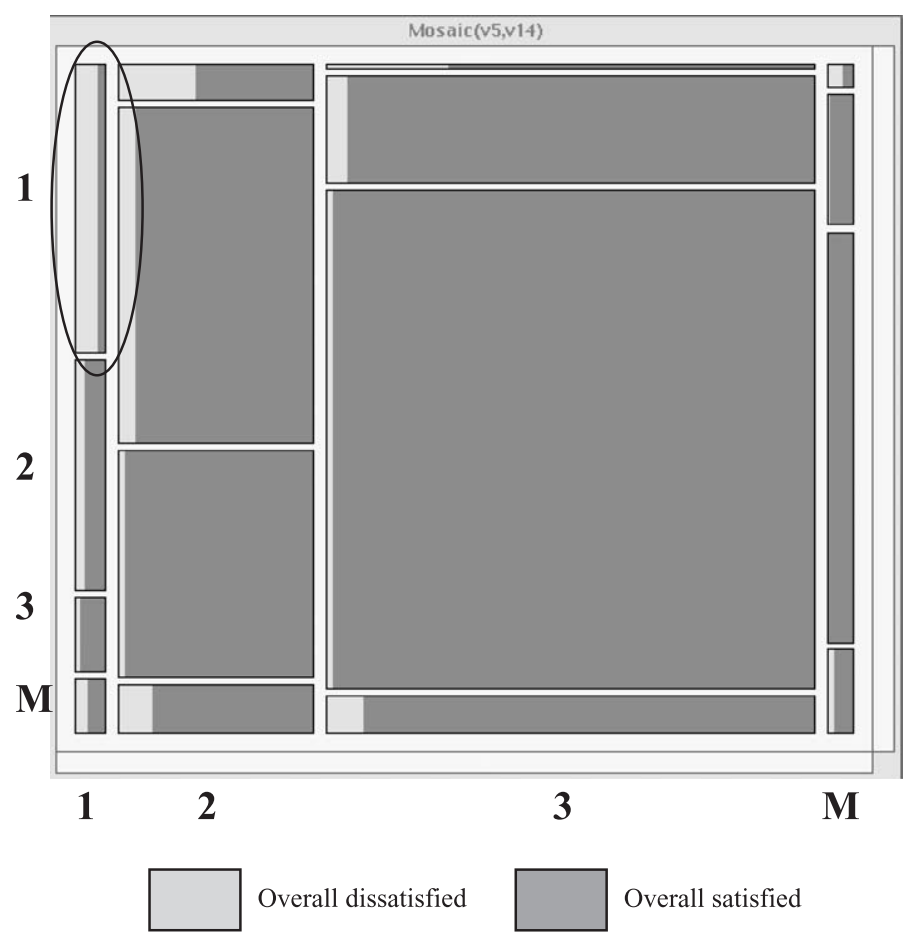

Fig. 4. The mosaic plot.

identify the regions with high overall dissatisfaction rates so that appropriate actions for dissatisfaction management can be taken.

With regard to heterogeneity, it can be seen that association rules represent densely populated areas in the multi-attribute instance space, such that they account for the heterogeneity present in the data. In fact, the reasons for being dissatisfied for an individual customer can easily be tracked by looking at the rules in the result set that fire (trigger) for that individual, such that for each individual the interactions of attributes that cause dissatisfaction are summarized in a limited set of rules (in our case, 3.6 rules on average per instance). Thus, heterogeneity in the sample is accounted for by the fact that different individuals are covered by other rules in the result set, making some (interactions of) attributes more important for some customers than for others. In contrast, in the case of logistic regression, an aggregate function is estimated that does not account for heterogeneity. For instance, according to the logistic regression model, dissatisfaction on questions 11 and 13 have the most negative impact on the overall level of satisfaction. However, 245 (of the 445) overall dissatisfied customers do not even mention dissatisfaction with these questions. However, they indicate dissatisfaction on other questions meaning that they have other reasons to be dissatisfied, which are not reflected by the aggregate logistic regression function. Consequently, we believe that the rule-based approach provides more accurate information about the reasons for dissatisfaction than the aggregate function-based approach. This is probably also why the mixture regression approach produces superior results in terms of predictive accuracy since it explicitly accounts for the heterogeneity present in the sample by estimating separate regression functions within each latent class.

Finally, the stability of the classification model for different sizes of the calibration and test should also be discussed. To this end, two major issues were investigated: structural stability (i.e. does the model look the same for different sample sizes) and stability with regard to the accuracy of the models (average accuracy and its standard deviation).

With regard to the structural stability, the logistic and mixture regression models tend to be more stable 
than classification trees (both C5.0 and CART), i.e. when the sample size of the calibration set is decreased, the classification tree models start to produce different models more quickly than the regression techniques. However, for both logistic regression and classification trees, the order in which the variables enter the model are different for different sample sizes. Furthermore, the model structure tends to be less stable for classification trees than for logistic regression when carrying out 10 -fold cross validation. In fact, mostly the same variables enter the classification tree, but only the order in which they enter the tree is different. The reason is 'variable masking', i.e. if one variable is highly correlated with another, then a small change in the calibration sample may shift the split from one variable to another. Yet, two splits of a node may be dissimilar but have almost the same goodness of split.

With respect to the stability of the accuracy however, both regression techniques and classification tree models produce stable results for 10 -fold cross validation. For all types of models, the variation of the average accuracy is small $(\sigma= \pm 0.3 \%)$. To summarize, although regression models tend to be structurally more stable on this data than classification tree models, this does not affect their average accuracy.

However, more importantly, the technique of association rules for partial classification is the most stable of all, both in terms of structural stability and accuracy, i.e. variation of the calibration sample size has almost no influence on the association ruleset model than on the other models. Moreover, it turned out that from the 29 patterns (Appendix A) included in the model based on the calibration set, 27 of them also appeared when using the test to discover a model, which indicates the high stability of the selected patterns. Table 2 summarizes the above conclusions.

\subsubsection{Determining group size of customers at risk}

The ROC convex hull graph solves the problem of comparing and determining the 'best' classification model in terms of overall classification performance. However, it does not solve the problem of determining the group size of customers at risk. In fact, for each technique, different parameter settings imply a different group size of customers at risk. For instance, increasing the cost of false negative classifications with classification trees, will imply the increase of the FP rate, which in turn increases the size of the group of customers at risk. In the case of association rule rulesets, the number of rules being retained in the ruleset determines the group size of customers at risk. Indeed, the more rules are retained (i.e. the more complex the ruleset model), the bigger the group of customers at risk becomes. This observation would imply that it is impossible to determine the correct group size of customers at risk. However, in the case of association rules, the minimum description length (MDL) principle provides a solution to this problem.

The MDL principle (Rissanen, 1978) is a suitable criterion to determine the optimal trade-off between model complexity (i.e. the number of rules in the ruleset) and the coverage of true positive instances. MDL is a general principle that is intended to encode the natural bias towards simpler theories that explain the same body of data. The idea was originally proposed in the context of a communication problem. The goal is to communicate a method (a classifier) that will enable the receiver to determine the class labels of the examples in the data set. It is assumed

Table 2

Overall comparison of different approaches

\begin{tabular}{|c|c|c|c|c|c|c|c|c|c|c|}
\hline & & \multicolumn{5}{|c|}{ Quantitative criteria } & \multicolumn{4}{|c|}{ Qualitative criteria } \\
\hline & & CAIC & $\mathrm{BIC}$ & Es & $R^{2}$ & $\overline{\mathrm{ROC}}$ & $\begin{array}{l}\text { Structural } \\
\text { stability }\end{array}$ & $\begin{array}{l}\text { Pred. } \\
\text { stability }\end{array}$ & $\begin{array}{l}\text { Ease of } \\
\text { interpretation }\end{array}$ & Heterogeneity \\
\hline \multirow[t]{4}{*}{ Complete classification } & Logistic regression & 726 & 709 & 1.0 & 0.19 & + & + & ++ & - & no \\
\hline & Mixture regression & 682 & 677 & 0.17 & 0.39 & ++ & + & ++ & -- & yes \\
\hline & CART & na & na & na & na & - & - & ++ & + & yes \\
\hline & $\mathrm{C} 5.0$ & na & na & na & na & -- & - & ++ & + & yes \\
\hline Partial classification & Association rules & na & na & na & na & + & ++ & ++ & ++ & yes \\
\hline \multicolumn{2}{|c|}{ Company's current procedures } & na & na & na & na & na & na & na & ++ & yes \\
\hline
\end{tabular}

$\mathrm{na}=$ not applicable, $++=$ strongly superior, $+=$ slightly superior, $-=$ slightly inferior, $--=$ strongly inferior. 
that a sender has the entire set of examples, while a receiver has the examples without their class labels. The sender needs to convey the proper class labelling of the example set to the receiver. The sender must essentially choose the shortest description for specifying the classes. Now in the case of the null model, the sender must simply transmit the classes of the examples in a sequence, but no model code. Thus, the sender transmits $N$ messages, each being a coded class label and according to information entropy, this will cost $-\log _{2}$ (\#examples) of bits. In the case where a model (theory) is built and it needs to be transmitted, the model should represent a theory for the receiver to determine the class label of the examples that are covered by the theory. The class labels of the examples that are not covered by the theory still need to be transmitted and encoded. The total amount of information needed to encode this is then represented by the MDL. Thus, in our partial classification model, by increasing the number of rules (i.e. building a more complex theory), the number of false negative classifications (i.e. errors) will decrease. Thus, the more rules that are included, the more positive instances are correctly classified, and the fewer errors are made on positive instances. In contrast, by simplifying the model, i.e. by including fewer rules, more errors will be made on positive instances. The total amount of bits needed to transmit the model and the examples is then,

$$
\left.\mathrm{MDL}=-\log _{2}(\text { rules })-\log _{2} \text { (false negative errors }\right) .
$$

Furthermore, this optimum also entails a particular group size of customers at risk. Table 3 shows that the optimum number of rules in the association rule ruleset equals to 29, which entails a group size of customers at risk of 733 , i.e. $10.0 \%$ of the total customer population.

In the case of logistic regression or discriminant analysis, there is no such criterion that can be used to determine the optimal complexity of the model and thus the group size of customers at risk cannot be inferred. This is certainly a disadvantage when compared with association rules.

\section{Conclusion}

In this study, we have compared two different methodological approaches to the identification of customers at risk, i.e. complete versus partial classification. The objective of complete classification models is to discriminate between the target classes and to cover all classes and all instances of a class whereas the objective of partial classification models is to find a good description of the target class, i.e. to find rules that are individually accurate but that do not necessarily cover all classes or all instances of a class. More specifically, C5.0, CART, logistic regression, mixture regression, and association rule rulesets were evaluated and the results compared on different quantitative and qualitative criteria. The ROC convex hull graph enables comparison of different types of classification techniques under different misclassification costs and class distributions. Although for this study association rule rulesets do not outperform regressionbased techniques, it produces better predictive results when compared with classification trees, such as CART and C5.0.

Furthermore, there are three important managerial advantages of using association rule rulesets. First of all, the evaluation of association rules enables detailed interpretation of frequently co-occurring service level (dis)satisfactions whereas such subtle interactions cannot be inferred from a function in the case of regression-based techniques. It has been shown that one can easily make an interpretation of such interactions by means of mosaic plots. From the example given it could be inferred that if customers agree with the statement that staff do not reserve sufficient time for them and if customers agree with the statement that staff are not flexible enough to solve their questions, then there is a very high

Table 3

MDL for different sizes of the AR set

\begin{tabular}{lllllllllllll}
\hline & 1 & 10 & 20 & 28 & 29 & 30 & 40 & 50 & 60 & 70 & 80 & 90 \\
\hline MDL & -11.7 & -15.3 & -16.1 & -16.2 & -16.3 & -16.1 & -14.9 & -14.6 & -14.2 & -13.8 & -13.6 & -10.4 \\
\hline
\end{tabular}


probability of these customers being dissatisfied. These interaction patterns clearly show specific topics for (dis)satisfaction management. Secondly, the minimum description length principle provides a metric to determine the group size of customers at risk, as a function of the complexity of the generated rulesets, whereas for the regression techniques, there is no such metric available. In the latter case, the group size of customers at risk might at best stay a good guess or be determined by an heuristic such as the number of people being dissatisfied with a certain service component. Thirdly, internal validation of the association rule rulesets on data from the next year's survey demonstrates the stability of the rulesets. In fact, $98 \%$ of the association rules in 1996 exactly occurred again in the ruleset model for 1997, indicating its structural stability. Throughout these 2 years, the rules that determine customers at risk stayed the same, whether there is a change in the size of the group of customers at risk as such, due to marketing action being taken. Finally, the identification of customers at risk by association rule rulesets can be seen as an valid early warning signal for those customers that might have a higher propensity to leave than others and are somewhat less willing to stay in a relationship.

\section{Acknowledgements}

This research is supported by a grant from the Fund for Scientific Research in Flanders to Tom Brijs. We gratefully thank the editor and the two anonymous reviewers for their insightful and constructive comments on earlier drafts of this article.

\section{Appendix A}

Overview of 29 most interesting rules for dissatisfaction, together with their statistical properties, and ranked in descending order of interestingness.

$s=$ percentage support of the rule within group of dissatisfied customers

$c=$ confidence of the rule (see Section 2.2.1)

$i=$ interestingness of the rule (see formula 1 in

Section 3.3.1)
New items that have not yet appeared in higher ranked rules are printed in bold.

1. IF personal treatment by personnel $=$ very dissatisfied AND clear explanation by the $\mathbf{s t a f f}=$ very dissatisfied THEN overall dissatisfied $(s=20 \% ; c=76 \% ; i=0.92)$

2. IF staff reserve sufficient time for $\mathbf{m e}=$ very dissatisfied AND staff are flexible to solve my questions $=$ very dissatisfied THEN overall dissatisfied $(s=23 \% ; c=75.5 \% ; i=0.91)$

3. IF clear explanation by the $\operatorname{staff}=$ very dissatisfied AND staff are flexible to solve my questions $=$ very dissatisfied THEN overall dissatisfied $(s=20.5 \% ; c=72 \% ; i=0.91)$

4. IF personal treatment by personnel = very dissatisfied AND staff are flexible to solve my questions $=$ very dissatisfied THEN overall dissatisfied $(s=23.6 \% ; c=70 \% ; i=0.91)$

5. IF staff give sufficient explanation $=$ very dissatisfied AND staff are flexible to solve my questions $=$ very dissatisfied THEN overall dissatisfied $(s=27 \% ; c=69.5 \% ; i=0.91)$

6. IF staff give sufficient explanation = very dissatisfied AND staff reserve sufficient time for me $=$ very dissatisfied THEN overall dissatisfied $(s=23.6 \% ; c=68 \% ; i=0.91)$

7. IF staff reserve sufficient time for me $=$ very dissatisfied AND personal treatment by personnel $=$ very dissatisfied THEN overall dissatisfied $(s=23.4 \% ; c=68 \% ; i=0.90)$

8. IF staff give sufficient explanation $=$ very dissatisfied AND personal treatment by personnel $=$ very dissatisfied THEN overall dissatisfied $(s=27.6 \% ; c=65 \% ; i=0.90)$

9. IF staff give sufficient explanation $=$ very dissatisfied AND clear explanation by the staff $=$ very dissatisfied THEN very dissatisfied $(s=23.6 \% ; c=64 \% ; i=0.90)$

10. IF staff are flexible to solve my questions = very dissatisfied AND I receive sufficient information by mail=very dissatisfied THEN very dissatisfied $(s=23.6 \% ; c=63.2 \% ; i=0.90)$

11. IF staff reserve sufficient time for $m e=$ very dissatisfied AND I receive sufficient information by mail $=$ very dissatisfied THEN very dissatisfied $(s=20.2 \% ; c=60.8 \% ; i=0.90)$

12. IF clear explanation by the staff= very dissatisfied AND I receive sufficient information by mail $=$ very dissatisfied THEN very dissatisfied $(s=20.2 \% ; c=59.2 \% ; i=0.89)$

13. IF staff give sufficient explanation $=$ very dissatisfied AND the bank's investment advice is sound $=$ very dissatisfied THEN very dissatisfied $(s=22.5 \% ; c=58.5 \% ; i=0.89)$

14. IF staff spontaneously speak about new service possibilities $=$ very dissatisfied AND staff are flexible to solve my questions $=$ very dissatisfied THEN very dissatisfied $(s=29.2 \% ; c=56.8 \% ; i=0.89)$

15. IF one has to queue for a long time in the bank office $=$ very dissatisfied AND staff are flexible to solve my questions = very dissatisfied THEN very dissatisfied $(s=23.4 \% ; c=54.5 \% ; i=0.89)$

16. IF staff give sufficient explanation $=$ very dissatisfied AND I receive sufficient information by mail $=$ very dissatisfied THEN very dissatisfied $(s=31 \% ; c=54.3 \% ; i=0.88)$

17. IF staff give sufficient explanation = very dissatisfied AND opening hours of the bank suit me well $=$ very dissatisfied THEN very dissatisfied $(s=24.5 \% ; c=53.9 \% ; i=0.88)$ 
18. IF one has to queue for a long time in the bank office $=$ very dissatisfied

AND clear explanation by the staff $=$ very dissatisfied

THEN very dissatisfied $(s=21.8 \% ; c=53.8 \% ; i=0.88)$

19. IF staff spontaneously speak about new service possibilities $=$ very dissatisfied

AND clear explanation by the staff $=$ very dissatisfied

THEN very dissatisfied $(s=25.4 \% ; c=53.6 \% ; i=0.88)$

20. IF staff reserve sufficient time for $m e=$ very dissatisfied AND staff spontaneously speak about new service possibilities $=$ very dissatisfied

THEN very dissatisfied $(s=26.1 \% ; c=52.7 \% ; i=0.88)$

21. IF personal treatment by personnel $=$ very dissatisfied

AND I receive sufficient information by mail $=$ very dissatisfied

THEN very dissatisfied $(s=25.4 \% ; c=52.6 \% ; i=0.88)$

22. IF staff reserves sufficient time for me $=$ very dissatisfied AND one has to queue for a long time in the bank office $=$ very dissatisfied

THEN very dissatisfied $(s=21.4 \% ; c=48.5 \% ; i=0.87)$

23. IF staff give sufficient explanation $=$ very dissatisfied

AND staff spontaneously speak about new service possibilities $=$ very dissatisfied

THEN very dissatisfied $(s=37.5 \% ; c=47.7 \% ; i=0.87)$

24. IF staff spontaneously speak about new service possibilities $=$ very dissatisfied

AND staff have sufficient discretion $=$ very dissatisfied THEN very dissatisfied $(s=23.6 \% ; c=46.5 \% ; i=0.87)$

25. IF staff give sufficient explanation $=$ very dissatisfied AND one has to queue for a long time in the bank office $=$ very dissatisfied

THEN very dissatisfied $(s=28.3 \% ; c=46.1 \% ; i=0.86)$

26. IF staff give sufficient explanation = very dissatisfied AND the bank's investment advice is sound $=$ no opinion THEN very dissatisfied $(s=20.9 \% ; c=41.5 \% ; i=0.85)$

27. IF staff spontaneously speak about new service possibilities $=$ very dissatisfied

AND personal treatment by personnel $=$ very dissatisfied THEN very dissatisfied $(s=33 \% ; c=40.3 \% ; i=0.84)$

28. IF important financial issues are discussed in a separate room $=$ very dissatisfied

AND staff spontaneously speak about new service possibilities = very dissatisfied

THEN very dissatisfied $(s=20.5 \% ; c=40 \% ; i=0.84)$

29. IF staff give sufficient explanation $=$ very dissatisfied

AND the bank office gives a slovenly impression= very satisfied

THEN very dissatisfied $(s=22.7 \% ; c=38.1 \% ; i=0.83)$

\section{Appendix B. The Apriori algorithm}

The Apriori algorithm (Agrawal \& Srikant, 1994) consists of two phases: frequent itemset generation and association rule generation. The first phase is computationally the most demanding and is based on a levelwise search principle. This principle states that an itemset can only be frequent if all of its subsets are also frequent.

The algorithm, presented below, makes use of two itemset collections, i.e. the candidate itemsets $(C)$ and the frequent itemsets $(F)$. Candidate itemsets are itemsets of size $k$ (i.e. $k$-itemsets) that are potential candidates for being frequent, but for which their frequency must be checked against the database. Those candidate itemsets that, after verification against the database indeed turn out to be frequent, are added to the list of frequent itemsets. The algorithm starts with putting all single itemsets (1-itemsets) into $C$ and calculating their support against the database. All 1-itemsets that turn out to be frequent $\left(F^{\prime}\right)$ are then added to the collection of frequent 1itemsets $(F)$. Subsequently, candidate itemsets of size 2 (2-itemsets) are generated from all frequent 1-itemsets. The algorithm is then applied a second time, now verifying the support of the candidate 2-itemsets against the database, and adding all frequent 2 -itemsets to $F$. This levelwise process is continued until no candidate itemsets can be generated anymore, or all candidate itemsets of size $k$ turn out to be nonfrequent. The output of the algorithm is thus all frequent itemsets in the data.

Phase 1: Frequent itemset generation

1. $C:=\{\{A\} \mid A \in R\}$;

2. $F:=\varnothing$;

3. $k:=1$;

4. while $C \neq \varnothing$ do

5. $F^{\prime}:=$ the sets $X \in C$ that are frequent;

6. add $F^{\prime}$ to $F$;

7. $C$ : =sets $Y$ of size $k+1$ such that each subset $W$ of $Y$ of size $k$ is frequent;

8. $k:=k+1$;

9. output of all frequent itemsets.

Given all frequent itemsets in the data (see algorithm for frequent itemset generation above), the second phase of the Apriori algorithm consists of generating association rules from these frequent itemsets. Basically, for all frequent itemsets this involves testing whether the rule antecedent $\Rightarrow$ consequent holds with sufficient confidence. This can be calculated fairly easily. For instance, let the 4-itemset $A B C D$ be frequent, then for the rule $A B C \Rightarrow D$ to 
be withheld, it must be verified whether the rule has sufficient confidence by checking whether sup$\operatorname{port}(A B C D) / \operatorname{support}(A B C) \geq \operatorname{minimum}$ confidence threshold.

Phase 2: Association rule generation

1. For all large itemsets $l \in F$ do

2. for all subsets $a$ of $l$ do

3 . verify confidence of $a \Rightarrow(l-a)$

4. output all $a \Rightarrow(l-a) \geq$ minimum confidence.

\section{References}

Agrawal, R., Imielinski, T., \& Swami, A. (1993). Mining association rules between sets of items in large databases. In P. Buneman, \& S. Jajodia (Eds.), Proceedings of the ACM SIGMOD conference on management of data (pp. 207-216). Washington, DC: ACM Press.

Agrawal, R., \& Srikant, R. (1994). Fast algorithms for mining association rules. In J. B. Bocca, M. Jarke, \& C. Zaniolo (Eds.), Proceedings of the 20th international conference on very large databases (pp. 487-499). Santiago, Chile: Morgan Kaufmann.

Aitkin, M., \& Rubin, D. (1985). Estimation and hypothesis testing in finite mixture models. Journal of the Royal Statistical Society $B, 47,67-75$.

Akaike, H. (1974). A new look at statistical model identification. IEEE Transactions on Automatic Control, AC-19, 716-723.

Ali, K., Manganaris, S., \& Srikant, R. (1997). Partial classification using association rules. In D. Heckerman, H. Mannila, D. Pregibon, \& R. Uthurusamy (Eds.), Proceedings of the third international conference on knowledge discovery and data mining (pp. 115-118). Newport Beach, CA: AAAI Press.

Anand, S. S., Hughes, J. G., Bell, D. A., \& Patrick, A. R. (1997). Tackling the cross-sales problem using data mining. In H. -J. Lu, H. Liu, \& H. Motoda (Eds.), Proceedings of the first PacificAsia conference on knowledge discovery and data mining (pp. 331-343). Singapore: World Scientific Pub.

Anderson, E. W. (1998). Customer satisfaction and word-of-mouth. Journal of Service Research, 1(1), 1-14.

Anderson, E. W., \& Mittal, V. (2000). Strengthening the satisfaction-profit chain. Journal of Service Research, 3(2), 107-120.

Anderson, E. W., \& Sullivan, M. W. (1993). The antecedents and consequences of customer satisfaction for firms. Marketing Science, 12(2), 125-143.

Berry, L. L. (1995). Relationship marketing of services - growing interest, emerging perspectives. Journal of the Academy of Marketing Science, 23(4), 236-245.

Bloemer, J. M. M., \& Kasper, J. D. P. (1995). The complex relationship between consumer satisfaction and brand loyalty. Journal of Economic Psychology, 16, 311-329.

Bolton, R. N. (1998). A dynamic model of the duration of the customer's relationship with a continuous service provider: the role of satisfaction. Marketing Science, 17(1), 45-65.
Bowman, D., \& Narayandas, D. (2001, August). Managing customer-initiated contacts with manufacturers: the impact on share of category requirements and word-of-mouth behavior. Journal of Marketing Research, 38, 281-297.

Bozdogan, H. (1987). Model selection and Akaike's information criterion (AIC): the general theory and its analytical extensions. Psychometrika, 52, 345-370.

Breiman, L., Friedman, J. H., Olshen, R. A., \& Stone, C. J. (1984). Classification and regression trees. California: Wadsworth.

Day, R. L. (1977). Toward a process model of consumer satisfaction. In H. K. Hunt (Ed.), Conceptualization and measurement of consumer satisfaction and dissatisfaction (pp. 153-183). Bloomington, IN: Marketing Science Institute.

Dver, A. (2001). Real-time customer data. . that's useful for retention. Bank Marketing, 33, 32-35.

Eisenblätter, D., \& Bozdogan, H. (1988). Two-stage multi-sample cluster analysis. In H. H. Bock (Ed.), Classification and related methods of data analysis (pp. 91-96). Amsterdam: Elsevier.

Enew, C. T., \& Brinks, M. R. (1996). The impact of service quality and service characteristics on customer retention: small business and their banks in the UK. British Journal of Management, 7, 219-230.

Ganesh, J., Arnoldt, M. J., \& Reynolds, K. E. (2000, July). Understanding the customer base of service providers: an examination of the differences between switchers and stayers. Journal of Marketing, 64, 65-87.

Garfein, R. (1987). Evaluating the impact of customer service delivery systems. Marketing Review, 42, 11-15.

Gensch, D. H. (1984). Targeting the switchable industrial customer. Marketing Science, 3(1), 41-54.

Hirschman, A. O. (1970). Exit, voice, loyalty: responses to decline in firms, organizations and states. Cambridge: Harvard Univ. Press.

Homburg, C., \& Giering, A. (2001). Personal characteristics as moderators of the relationship between customer satisfaction and loyalty - an empirical analysis. Psychology and Marketing, $18,43-66$.

Jones, M. A., Mothersbaugh, D. L., \& Beatty, S. E. (2000). Switching barriers and repurchase intentions in services. Journal of Retailing, 76(2), 259-274.

Oliver, R. L. (1980, November). A cognitive model of the antecedents and consequences of satisfaction decisions. Journal of Marketing Research, 17, 460-469.

Oliver, R. L. (1997). Satisfaction. A behavioral perspective on the consumer. New York: McGraw Hill.

Oliver, R. L., \& Swan, J. E. (1989a, April). Consumer perceptions of interpersonal equity and satisfaction in transactions: a field survey approach. Journal of Marketing, 53, 21-35.

Oliver, R. L., \& Swan, J. E. (1989b, December). Equity and disconfirmation perceptions and influences on merchant and product satisfaction. Journal of Consumer Research, 16, 372-383.

Provost, F., \& Fawcett, T. (1997). Analysis and visualization of classifier performance: comparison under imprecise class and cost distributions. In D. Heckerman, H. Mannila, D. Pregibon, \& R. Uthurusamy (Eds.), Proceedings of the third international conference on knowledge discovery and data mining (pp. 43-46). Newport Beach, CA, USA: AAAI Press. 
Quinlan, J. R. (1993). C4.5: programs for machine learning. San Mateo, CA: Morgan Kaufmann.

Rissanen, J. (1978). Modeling by shortest data description. Automatica, 14, 465-471.

Schwarz, G. (1978). Estimating the dimension of a model. Annals of Statistics, 6, 461-464.

Swan, J. E., Trawick, I. F., \& Carroll, M. G. (1982). Satisfaction related to predictive, desired expectations: a field of study. In R. L. Day, \& H. K. Hunt (Eds.), New findings on consumer satisfaction and complaining (pp. 15-22). Bloomington, IN: University Press.
Viveros, M. S., Nearhos, J. P., \& Rothman, M. J. (1996). Applying data mining techniques to a health insurance information system. In T. M. Vijayaraman, A. P. Buchmann, C. Mohan, \& N. L. Sarda (Eds.), Proceedings of the 22nd VLDB conference (pp. 286-294). Mumbai (Bombay), India: Morgan Kaufmann.

Wedel, M., \& Kamakura, W. A. (1999). Market segmentation: conceptual and methodological foundations (2nd ed.). Dordrecht: Kluwer.

Zeithaml, V. A., Berry, L. L., \& Parasuraman, A. (1996, April). The behavioral consequences of service quality. Journal of Marketing, 60, 31-46. 